\title{
WSKY, a traditional Chinese decoction, rescues cognitive impairment associated with NMDA receptor antagonism by enhancing BDNF/ERK/CREB signaling
}

\author{
XIN GUO $^{1}$, ZHENG-HUA CHEN ${ }^{1}$, HUI-LING WANG ${ }^{1}$, ZHONG-CHUN LIU ${ }^{1}$, XIAO-PING WANG ${ }^{1}$, \\ BEN-HONG ZHOU ${ }^{2}$, CAN YANG $^{1}$, XUE-PING ZHANG ${ }^{1}$, LING XIAO ${ }^{1}$, CHANG SHU $^{1}$, \\ JIAN-XIN CHEN $^{1}$ and GAO-HUA WANG ${ }^{1}$
}

Departments of ${ }^{1}$ Psychiatry and ${ }^{2}$ Pharmacy, Renmin Hospital of Wuhan University, Wuhan, Hubei 430060, P.R. China

Received November 30, 2013; Accepted May 30, 2014

DOI: $10.3892 / \mathrm{mmr} .2014 .3086$

\begin{abstract}
Warm-supplementing kidney yang (WSKY) is an herbal prescription that has been used in Traditional Chinese Medicine for the treatment of psychiatric conditions. A previous study by our group found that WSKY significantly improved cognitive function of schizophrenia patients. In the present study, the effects of WSKY on cognitive function and their underlying mechanisms were investigated. WSKY was administered to an MK-801-induced rat model of chronic schizophrenia for 14 days. Memory performance was assessed using the Morris water maze (MWM) test. The expression of brain-derived neurotrophic factor (BDNF), activation of cAMP response element binding protein (pCREB/CREB) and activation of extracellular signal-regulated kinase (pERK/ERK) in the hippocampus was detected using western blot analysis. In the acquisition phase of the MWM test, the escape latency was significantly increased in the MK-801-treated group compared with the normal control group $(\mathrm{P}<0.01)$. Treatment with WSKY for 14 days at doses of 100 or $250 \mathrm{mg} / \mathrm{kg}$ rescued this cognitive impairment $(\mathrm{P}<0.05)$. In the probe test, $250 \mathrm{mg} / \mathrm{kg}$ WSKY treatment increased the time spent in the target quadrant $(\mathrm{P}<0.05)$ and number of platform crossings $(\mathrm{P}<0.01)$. Western blot analysis demonstrated that the levels of BDNF expression in the hippocampus of rats without behavioral tests were elevated following 14 days of WSKY treatment, and the effect of WSKY treatment on hippocampal BDNF expression was presented in an inverted U-shaped dose-response pattern.
\end{abstract}

Correspondence to: Professor Gao-Hua Wang, Department of Psychiatry, Renmin Hospital of Wuhan University, 238 Jiefang Road, Wuhan, Hubei 430060, P.R. China

E-mail: fingerling1012@gmail.com

Abbreviations: WSKY, warm-supplementing kidney yang

Key words: warm-supplementing kidney yang, schizophrenia, cognitive performance, MK-801, Morris water maze, brain-derived neurotrophic factor
The pERK1/2 in the hippocampus was significantly enhanced following $100 \mathrm{mg} / \mathrm{kg}(\mathrm{P}<0.01)$ and $250 \mathrm{mg} / \mathrm{kg}(\mathrm{P}<0.01) \mathrm{WSKY}$ treatment, while only $250 \mathrm{mg} / \mathrm{kg}$ WSKY increased the phosphorylation of CREB $(\mathrm{P}<0.01)$. The results of the present study indicated that WSKY enhances cognitive performance via the upregulation of BDNF/ERK/CREB signaling, and that WSKY has potential therapeutic implications for cognitive impairment of schizophrenia.

\section{Introduction}

Schizophrenia is characterized by positive symptoms, including delusions and hallucinations, negative symptoms such as avolation and flat affect, and cognitive impairments (1). The cognitive symptoms of schizophrenia often precede the occurrence of psychosis (2), and their treatment is considered an improved predictor of therapeutic outcome (3), in particular, cognitive dysfunction often remains resistant to treatment with current antipsychotics, even following remission of the psychosis (4). Consequently, developing novel compounds that demonstrate increased efficacy against cognitive dysfunction of schizophrenia is urgently required (5).

Warm-supplementing kidney yang (WSKY) is a herbal prescription that has been used for the treatment in psychiatric conditions in Traditional Chinese Medicine (TCM). According to TCM, schizophrenia is a syndrome caused by a yin-yang imbalance characterized by spleen and stomach weakness and inadequate kidney yin. Herbal prescriptions, such as WSKY or strong kidney yin, are considered to improve deficits associated with schizophrenia. Previously, it was reported that WSKY capsules in addition to risperidone significantly enhanced cognitive function, social function (6) and improved the quality of life (7) compared with the placebo added to risperidone. The present study focused on the mechanisms underlying WSKY's effects on cognition enhancement.

Brain-derived neurotrophic factor (BDNF) and its receptors and downstream cascade have been associated not only with neurodevelopment and neuroprotection, but also with synapse regulation, learning and memory (8). BDNF has a critical role in synaptic plasticity through $N$-methyl-D-aspartate (NMDA) receptor activation in the hippocampus (9). Hippocampal long 
term potentiation (LTP), which involves the strengthening of synapses as the result of binding of BDNF to the tropomyosin-related kinase $\mathrm{B}$ (TrkB) receptor, is responsible for learning and memory (10), and impairment as the result of lack of BDNF was found to be restored by reintroduction of BDNF. Furthermore, cognitive impairment observed in schizophrenia suggests that BDNF may be a potential biomarker candidate as its effect has been implicated in learning and memory (11).

In the present study, the Morris water maze (MWM) test was utilized to identify cognition improvement of WSKY in a rat model of schizophrenia. The expression of BDNF-related signaling molecules, including, brain-derived neurotrophic factor (BDNF), phosphorylated extracellular signal-regulated kinase (pERK) and phosphorylated cAMP response element binding protein (pCREB) expression in rat hippocampus was observed using western blotting analysis following administration of WSKY in a chronic manner. The results of the present study indicated that WSKY has potential therapeutic implications for cognitive impairment in schizophrenia and psychiatric diseases involving abnormal expression of BDNF.

\section{Materials and methods}

Animals. Male Sprague-Dawley rats (weighing 300-350 g, corresponding to $\sim 2$ months of age) were purchased from Wuhan University, Center of Experimental Animal (Wuhan, China). The rats were housed at four animals per cage and were provided with food and water ad libitum and maintained under a 12-h light/dark cycle (light on 07:30-19:30) at constant temperature $\left(23 \pm 1^{\circ} \mathrm{C}\right)$ and humidity $(60 \pm 10 \%)$. A 60 -min habituation period to the experimental room on the first day and $30 \mathrm{~min}$ on the following days preceded the behavioral experiments. The number of animals used in each group for behavioral tests is described in Table I. Animal treatment and maintenance were conducted in accordance with the Principles of Laboratory Animal Care (NIH publication no. 85-23, revised 1985) and the Animal Care and the use guidelines of Wuhan University (Wuhan, China). The present study was approved by the ethics committee of Wuhan University.

Materials. MK-801 and rabbit polyclonal anti-BDNF antibodies were purchased from Santa Cruz Biotechnology, Inc. (Santa Cruz, CA, USA). The rabbit monoclonal anti-phosphorylated ERK, rabbit monoclonal anti-ERK, rabbit monoclonal anti-phosphorylated CREB and rabbit monoclonal anti-CREB antibodies were purchased from Cell Signaling Technology, Inc., (Danvers, MA, USA). All of the other materials were of the highest grades available and were obtained from commercial sources.

Preparation of WSKY. WSKY consisted of 13 traditional Chinese herbs (Table II). All of the ingredients were purchased from Kang Sheng Pharmaceutical Co., Ltd. (Wuhan, China) and carefully authenticated by Dr. Ben-Hong Zhou (Department of Pharmacy, Renmin Hospital of Wuhan University, Wuhan, China). The voucher specimens were deposited at the Herbarium of the Renmin Hospital of Wuhan University: Aconitum Carmichaeli Debx WHUOPS2010-12, Herba Epimedii WHUOPS2010-10, Radix Morinda Officinalis WHUOPS2010-32, Cortex
Table I. Numbers of animals used in each group.

\begin{tabular}{lll}
\hline Test & Group and drug dose $(\mathrm{mg} / \mathrm{kg})$ & $\mathrm{n}$ \\
\hline MWM test & Control & 6 \\
(with MWM training) & Vehicle + MK-801 (0.05) & 8 \\
& WSKY (25)+ MK-801 (0.05) & 6 \\
& WSKY (100)+ MK-801 (0.05) & 8 \\
& WSKY (250) + MK-801 (0.05) & 7 \\
& WSKY (500) + MK-801 (0.05) & 8 \\
Western blot analysis & Control & 6 \\
(without MWM training) & WSKY (25) & 7 \\
& WSKY (100) & 6 \\
& WSKY (250) & 6
\end{tabular}

WSKY, warm-supplementing kidney yang; n, number of animals; IHC, immunohistochemistry; MWM, Morris water maze.

Cinnamomoi WHUOPS2010-24, Rhizoma Zingiberis WHUOPS2010-34, Radix Rehmanniae WHUOPS2010-28, Radix Glycyrrhizae WHUOPS2010-16, Radix Astragali WHUOPS2010-8, Pericarpium Citri Reticulatae WHUOPS2010-15, Fructus Amomi WHUOPS2010-7, Carapax Et Plastrum Testudinis WHUOPS2010-30, Radix Codonopsis Pilosulae WHUOPS2010-9 and Rhizoma Curculigins WHUOPS2010-33 (purchased from Kang Sheng Pharmaceutical Co. Ltd., Wuhan, China). The procedure of preparation of WSKY was performed in accordance with the method used in a previous study by our group (6). The volatile oil of Pericarpium Citri Reticulatae, Cortex Cinnamomoi and Rhizoma Zingiberis were extracted by steam distillation. Dregs of these three components were added to the other components for water extraction and ethanol precipitation extraction. Icariin [National Institute for the Control of Pharmaceutical and Biological Products (NICPBP), Beijing, China] was detected as an indicator of orthogonal test to determine the optimal conditions (Table III). According to these optimal conditions, all of the ingredients were firstly soaked in 11 times their volume of distilled water and boiled for $1 \mathrm{~h}$. Following filtration, the residue was added to distilled water ten times its volume again and boiled for $1 \mathrm{~h}$. The two filtrates were combined and concentrated to a liquid ratio of 1:1, 70\% alcohol precipitation extraction, then concentrated with a rotary evaporator and lyophilized (ALPHA1-4; Martin Christ Gefriertrocknungsanlagen GmbH, Osterode am Harz, Germany) to dry powder. The volatile oil was sprayed evenly onto the dry the extract powder. The final dried extract was $32.4 \%$ (w/w) of the weight of the raw herbs. For quality control of WSKY extraction, icariin, as a standard of quality control, was purchased from the NICPBP, and a high-performance liquid chromatography system (Agilent 1100; Agilent Technologies, Santa Clara, CA, USA) equipped with a diode array detector and a Venusil MP-C18 chromatographic column (4.6×250 mm, $5 \mu \mathrm{m}$; Bonna-Agela Technologies, Inc., Wilmington, DE, USA) was used for standardization of the herbal extract (Fig. 1). 
Table II. Ingredients and botanical/zoological origins of warm-supplementing kidney yang formula.

\begin{tabular}{lll}
\hline Pharmacological name & \multicolumn{1}{c}{ Botanical/zoological source/family } & \multicolumn{1}{c}{ Part used } \\
\hline Aconitum carmichaeli Debx & Aconitum carmichaeli Debx. & Daughter root (processed) \\
Radix Morinda officinalis & Morinda officinalis F.C. How & Dried root and rhizome \\
Herba Epimedii & Epimedium brevicornu Maxim. & Dried body \\
Rhizoma Curculigins & Curculigo orchioides Gaertn. & Dried root and rhizome \\
Cortex Cinnamomoi & Cinnamomum cassia (Nees \& T. Nees) J. Presl & Dried tender branch \\
Rhizoma Zingiberis & Zingiber officinale Rosc & Dried root and rhizome \\
Radix Codonopsis Pilosulae & Codonopsis pilosula (Franch.) Nannf & Dried root and rhizome \\
Radix Astragali & Astragalus abbreviatus Kar. \& Kir. & Dried root and rhizome \\
Radix Rehmanniae & Rehmannia glutinosa (Gaertn.) DC & Root (processed) \\
Pericarpium Citri Reticulatae & Citrus reticulata Blanco & Dried mature peel \\
Fructus Amomi & Amomum villosum Lour & Dried fruit \\
Radix Glycyrrhizae & Glycyrriza uralensis Fisch & 9 \\
Carapax Et Plastrum Testudinis & Chinemys reevesii (Gray) & Dried root and rhizome \\
\hline
\end{tabular}

Table III. Results of orthogonal test.

\begin{tabular}{|c|c|c|c|c|c|c|}
\hline $\begin{array}{l}\text { Experiment } \\
\text { number }\end{array}$ & $\begin{array}{l}\text { Water volume } \\
\text { (fold) }\end{array}$ & Heat-extraction (h) & $\begin{array}{c}\text { Ethanol } \\
\text { precipitation }(\%)\end{array}$ & $\begin{array}{c}\text { Ethanol } \\
\text { precipitation }(\mathrm{h})\end{array}$ & $\begin{array}{c}\text { Icariin } \\
\text { content }(\mathrm{mg} / \mathrm{g})\end{array}$ & $\begin{array}{c}\text { Extraction } \\
\text { rate }(\%)\end{array}$ \\
\hline 1 & 8 & 1 & 60 & 12 & 0.22 & 18.64 \\
\hline 2 & 8 & 1.5 & 70 & 24 & 0.20 & 18.99 \\
\hline 3 & 8 & 2 & 80 & 48 & 0.19 & 15.28 \\
\hline 4 & 10 & 1 & 70 & 48 & 0.26 & 21.87 \\
\hline 5 & 10 & 1.5 & 80 & 12 & 0.19 & 16.91 \\
\hline 6 & 10 & 2 & 60 & 24 & 0.15 & 21.36 \\
\hline 7 & 12 & 1 & 80 & 24 & 0.27 & 14.77 \\
\hline 8 & 12 & 1.5 & 60 & 48 & 0.13 & 19.39 \\
\hline 9 & 12 & 2 & 70 & 12 & 0.22 & 22.39 \\
\hline
\end{tabular}

Fold, water volume/ingredients volume.

Drug administration. WSKY or MK-801 was dissolved in $0.9 \%$ saline solution. In a pilot study, it was observed that a 14-day consecutive injection of MK-801 at $0.05 \mathrm{mg} / \mathrm{kg}$ induced impairment of the spatial memory in rats, as was observed in other studies (12). Based on these results, the effective dose of MK-801 $(0.05 \mathrm{mg} / \mathrm{kg})$ was adopted for further experiments. In addition, the effective dose of WSKY $(25,100,250$ or $500 \mathrm{mg} / \mathrm{kg}$, W/W) that was selected was roughly translated from the dosage of the WSKY capsule, according to a previous study based on the human-animal dose translation equation (13). The rats were orally administered WSKY for 14 days (Fig. 2B). During the behavioral sessions, the rats were subjected to WSKY treatment $2 \mathrm{~h}$ prior to the behavioral tests, and MK-801 (0.05 mg/kg, i.p.) was administered $30 \mathrm{~min}$ prior to the behavioral tests. The control group was orally administered $0.9 \%$ saline solution $2 \mathrm{~h}$ prior to the behavioral tests.

MWM test. The MWM test was conducted as previously described (12). The water maze consisted of a circular, $180 \mathrm{~cm}$ diameter and $45 \mathrm{~cm}$ deep, black tank filled with water at $22^{\circ} \mathrm{C}$ to a depth of $28 \mathrm{~cm}$. The testing room was maintained at $22^{\circ} \mathrm{C}$ and contained fixed extra-visual cues. A circular, $15 \mathrm{~cm}$ diameter escape platform composed of black plexiglas was placed in the pool $1 \mathrm{~cm}$ below the water surface.

The pool was divided into four equally sized quadrants (northeast, southeast, southeast and southwest). The platform was constantly placed in the 'Northeast' (Fig. 2A) halfway between the center and edge for all hidden platform testing. The animals were always examined in the same order at approximately the same time each day. On day one, the first trial was started from the starting point 'South'. The starting points varied over the days of training, and they were rotated clockwise, one-quarter of a turn per trial.

Assessment of behavioral performance was conducted with the assistance of EthoVision ${ }^{\mathrm{TM}}$ (Noldus Information Technology, Wageningen, The Netherlands) The rats received the first trial, four trials per day, over 14 consecutive days (Fig. 2B). For each trial, the rats were placed into the water gently facing the wall. 


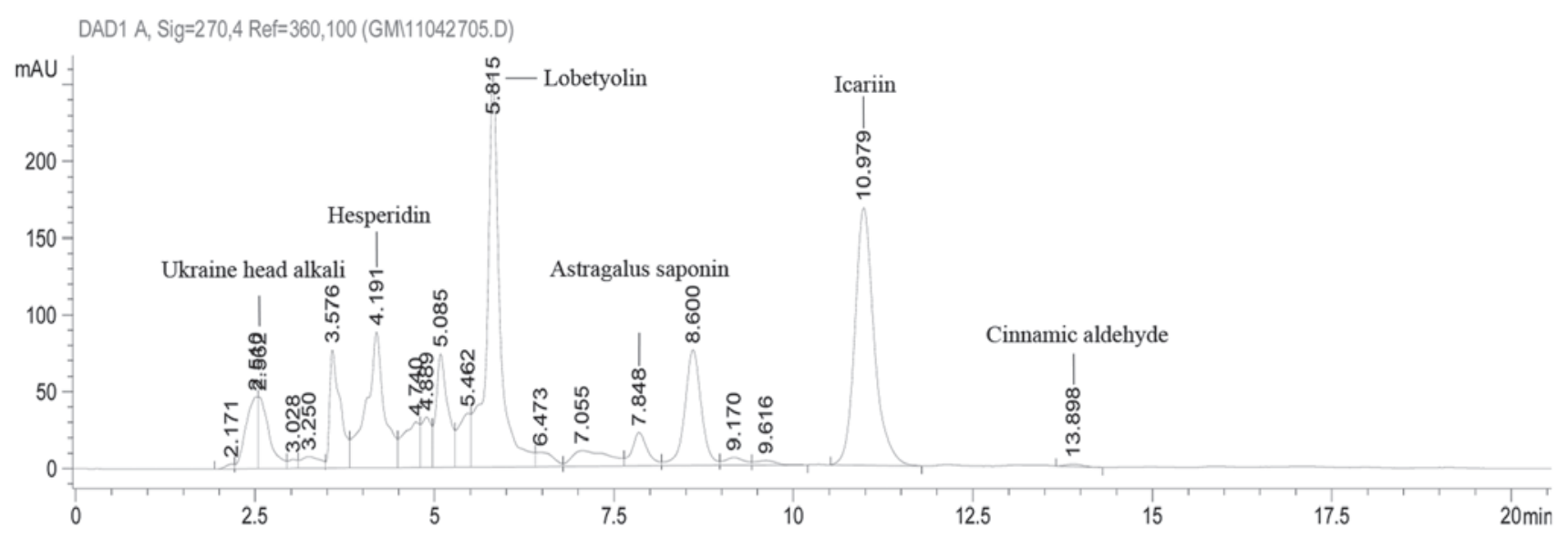

Figure 1. Analytical high-performance liquid chromatography of the warm-supplementing kidney yang extract. Icariin was used as a standard of quality control, the mobile phase consisted of acetonitrile - water (30:70), the flow rate was $1.0 \mathrm{ml} / \mathrm{min}$, the detection wavelength $279 \mathrm{~nm}$ and column temperature $25^{\circ} \mathrm{C}$. The retention time of icariin was $11 \mathrm{~min}$ and the relative standard deviation was $0.45 \%$ in the precision test.

The rats were then permitted to swim until they reached the escape platform in a maximum of $60 \mathrm{sec}$. Once on the platform, the rats remained there for $30 \mathrm{sec}$ prior to being removed for an inter-trial interval of $15 \mathrm{~min}$. Probe trials were conducted $24 \mathrm{~h}$ following the last hidden platform training. Each animal was allowed to swim for $30 \mathrm{sec}$ starting from the Southwest position.

Western blot analysis. For the preparation of western blot samples, the rats without behavioral training were sacrificed $1 \mathrm{~h}$ following the last administration of WSKY, and their isolated hippocampal tissues were analyzed by $4-10 \%$ SDS-PAGE (Beyotime Institute of Biotechnology, Haimen, China) and blotted onto polyvinylidene fluoride membranes. The western blots were incubated for $1 \mathrm{~h}$ with $5 \%$ skimmed milk, and then with rabbit anti-pERK1/2 (1:2,500 dilution), rabbit anti-ERK1/2 (1:5,000 dilution), rabbit anti-pCREB (1:2,500 dilution), rabbit anti-CREB (1:5,000 dilution) or rabbit anti-BDNF (1:1,000 dilution) antibodies overnight at $4^{\circ} \mathrm{C}$ with gentle agitation. The secondary antibodies were added and incubated with gentle rocking for $45 \mathrm{~min}$ at room temperature prior to washing of the membranes as described above. To analyze the relative protein quantity, $\beta$-actin was used as a loading control.

Statistical analysis. Values are expressed as the mean \pm standard error of the mean. $\mathrm{P}<0.05$ was considered to indicate a statistically significant difference between values. For the data from the MWM task, the treatment differences in the escape latency or average swim speed were analyzed using repeated measures analysis of variance (ANOVA) with a least significant difference (LSD) test as a post-hoc test. The time spent in the target quadrant (\%) and the number of platform crossings during the probe test was analyzed by one-way ANOVA with LSD test as a post-hoc test for each group. The data obtained from the western blot analysis of hippocampal tissues from rats were analyzed by one-way ANOVA with LSD test as a post-hoc test. Statistical analysis was performed using SPSS 17.0 for Windows (IBM, Armonk, NY, USA).

\section{Results}

Effect of WSKY on spatial learning and memory in the MWM test. MWM is a test for assessing spatial learning and memory (SLM) to locate a submerged escape platform (14). The present study examined whether WSKY ameliorated the MK-801-induced SLM impairment by using the MWM test (Fig. 2). Spatial learning was assessed by measuring the time taken to escape from the water to a hidden platform (escape latency).

The experimental schedule is demonstrated in Fig. 2B. Repeated-measure ANOVA revealed a significant delay effect on escape latency $\left(\mathrm{F}_{4,167}=600.837 ; \mathrm{P}<0.01\right)$, indicating that, across the groups, the rats improved their spatial learning effectively with increased training. The MK-801-injected rats demonstrated significant spatial reference memory function impairment during the training days compared with the control group $\left(\mathrm{F}_{1,23}=502.759, \mathrm{P}<0.001\right)$, while the performance of MK-801-treated rats was significantly improved by consecutive training $\left(\mathrm{F}_{4,115}=37.14, \mathrm{P}<0.01\right)$. There was also a significant treatment effect on the escape latency; WSKY treatment of schizophrenic animals at doses of 25, 100 and $250 \mathrm{mg} / \mathrm{kg}$ demonstrated significant SLM performance improvement compared with the MK-801-treated group (WSKY $25 \mathrm{mg} / \mathrm{kg}$ group, $\mathrm{F}_{1,27}=10.248, \mathrm{P}<0.05$; WSKY $100 \mathrm{mg} / \mathrm{kg}$ group, $\mathrm{F}_{1,27}=206.159, \mathrm{P}<0.01$; WSKY $250 \mathrm{mg} / \mathrm{kg}$ group, $\mathrm{F}_{1,27}=273.908, \mathrm{P}<0.01$, respectively, compared with the MK-801 group), which indicated that WSKY ameliorated the SLM impairment induced by MK-801 injection. Notably, WSKY at a dose of $500 \mathrm{mg} / \mathrm{kg}$ demonstrated no significant difference in training compared with that in the MK-801 group $\left(F_{1,27}=0.622, P>0.05\right.$; Fig. $\left.2 \mathrm{C}\right)$. However, there was no significant difference in the swimming speed between the groups (Fig. 2D). This result was different from that of a previous study (12), which is possibly associated with the different visual cues settings of the MWM test used in the present study.

Furthermore, the effects of WSKY on spatial memory formation were investigated. The probe trials were conducted to assess the spatial memory at $24 \mathrm{~h}$ following the final 
A

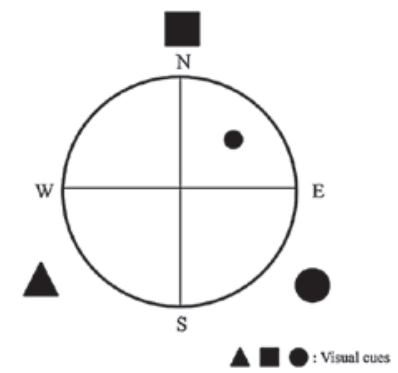

C

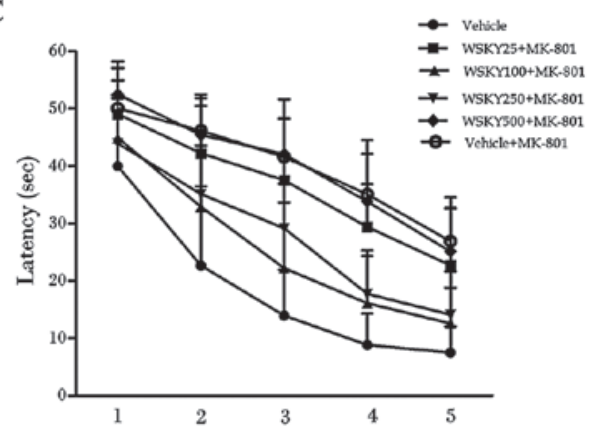

E

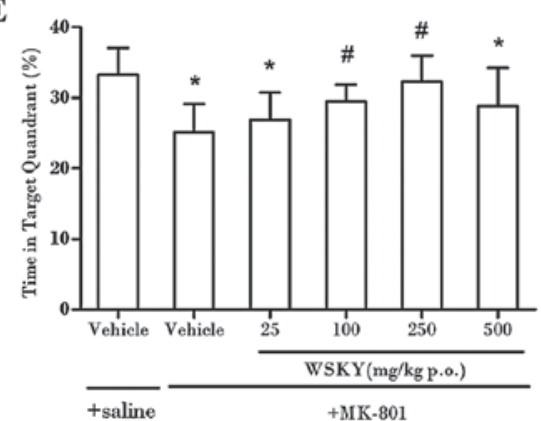

B

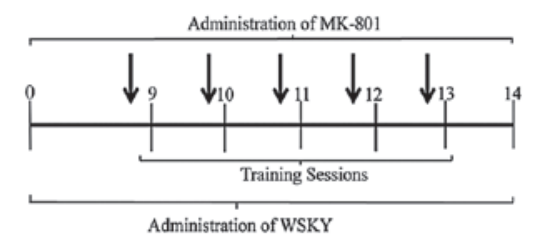

D

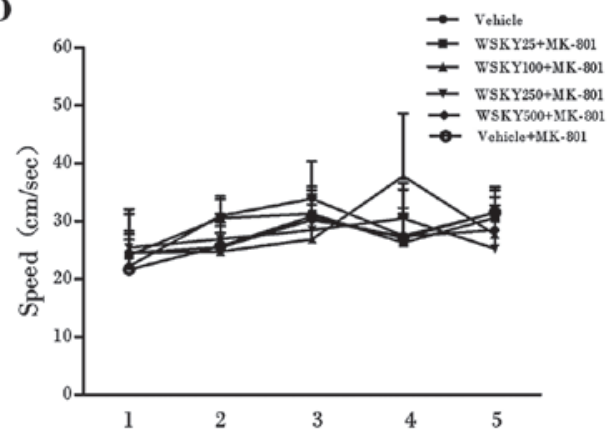

F

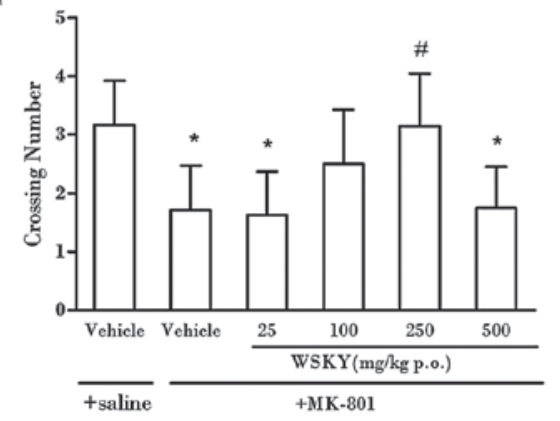

Figure 2. Effect of WSKY on the SLM of MK-801-treated rats in the MWM test. (A) The apparatus of the MWM test included a $180 \mathrm{~cm}$ diameter pool, a $15 \mathrm{~cm}$ diameter hidden platform and extra visual cues. (B) Four training trails of the MWM test per day were conducted for five consecutive sessions, probe trail was performed $24 \mathrm{~h}$ following the last training session. (C) The mean escape latency of each group to locate the hidden platform was recorded and analyzed during the MWM test. MK-801-injected rats demonstrated significant spatial reference memory function impairment during training days compared with the control group, while WSKY treatment at dose of 25,100 and $250 \mathrm{mg} / \mathrm{kg}$ ameliorated the SLM impairment induced by MK-801 injection; however, WSKY treatment at dose of $500 \mathrm{mg} / \mathrm{kg}$ did not. (D) There was no significant difference in the swimming speed in each group. (E) WSKY improved the SLM of MK-801-treated rats in the probe trail. WSKY treatment at doses of 100 and $250 \mathrm{mg} / \mathrm{kg}$ led to a significant increase in the time spent in the target quadrant and (F) $250 \mathrm{mg} / \mathrm{kg}$ treatment led to a significant increase in the number of platform crossings when compared with the MK-801 treated group. Values are presented as the mean \pm standard error of the mean. ${ }^{*} \mathrm{P}<0.05,{ }^{* *} \mathrm{P}<0.01$ vs. the control group; ${ }^{*} \mathrm{P}<0.05,{ }^{\# \#} \mathrm{P}<0.01$ vs. the MK-801-treated group. WSKY, warm-supplementing kidney yang; SLM, spatial learning and memory; MWM, Morris water maze.

training. As demonstrated in Fig. 2E, the 100 and $250 \mathrm{mg} / \mathrm{kg}$ WSKY-treated groups spent more time in the target quadrant of the pool in which the hidden platform was previously located than the MK-801 group (WSKY $100 \mathrm{mg} / \mathrm{kg}: 29.1 \pm 4.9$ vs. MK-801: 21.5 $\pm 2.5, \mathrm{P}=0.039, \mathrm{P}<0.01$; WSKY $250 \mathrm{mg} / \mathrm{kg}$ : $32.31 \pm 3.65$ vs. $\mathrm{MK}-801: 21.5 \pm 2.5, \mathrm{P}=0.002, \mathrm{P}<0.01)$. In addition, the $250 \mathrm{mg} / \mathrm{kg}$ WSKY treatment group demonstrated a significantly increased number of platform crossings when compared with the MK-801 treated group (WSKY $250 \mathrm{mg} / \mathrm{kg}$, $2.33 \pm 0.58$ vs. MK- $801,0.67 \pm 0.58$; P $<0.01$; Fig. $2 \mathrm{~F}$ ). This indicated that WSKY improved the SLM of rats treated with MK-801.

Effect of WSKY on BDNF expression in the hippocampus. BDNF has a key role in participating in the processes of synaptic plasticity and learning by enhancing long-term potentiation (LTP) (8), which is regarded as the cellular basis of learning and memory in the hippocampus. BDNF levels have previously been positively associated with cognitive impairment of schizophrenia patients $(15,16)$. To determine whether BDNF was involved in the cognitive enhancement by WSKY, the expression of BDNF in the hippocampal region of rats without MWM test was detected by western blot analysis. It was identified that treatment of rats with 100, 250 or $500 \mathrm{mg} / \mathrm{kg}$ WSKY for 14 days resulted in an increase in BDNF expression levels (WSKY 100 mg/kg, P<0.001; WSKY $250 \mathrm{mg} / \mathrm{kg}, \mathrm{P}<0.001$; WSKY $500 \mathrm{mg} / \mathrm{kg}, \mathrm{P}<0.05$; respectively, compared with the vehicle-treated group), while the $25 \mathrm{mg} / \mathrm{kg}$ WSKY treatment group revealed no significant difference with the vehicle-treated group $(\mathrm{P}=0.915, \mathrm{P}>0.05)$. The of BDNF 

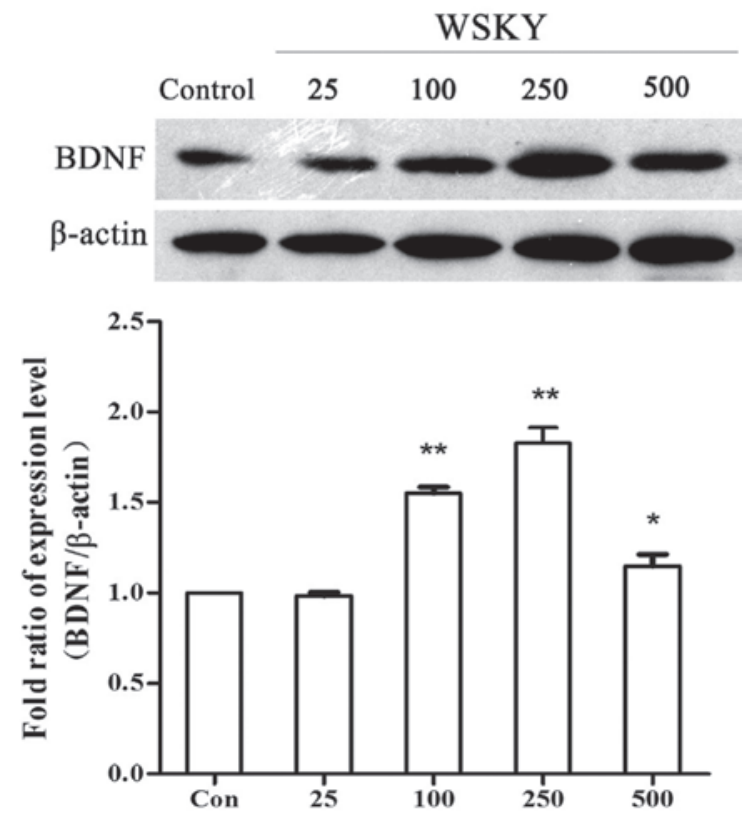

Figure 3. Effect of WSKY on BDNF protein expression in rat hippocampus without training. Treatment of rats with 100,250 or $500 \mathrm{mg} / \mathrm{kg}$ WSKY for 14 days resulted in an increase in BDNF expression. Western blotting ( $30 \mu \mathrm{g}$ protein/lane) with anti-BDNF antibody revealed that BDNF protein expression in the hippocampus demonstrated an inverted U-shaped dose-response pattern. The blot represents typical results from three independent experiments. Densitometric data are presented as the mean \pm standard error of the mean. ${ }^{*} \mathrm{P}<0.05,{ }^{* *} \mathrm{P}<0.01$ vs. the control group. WSKY, warm-supplementing kidney yang; BDNF, brain-derived neurotrophic factor.

protein expression levels in the hippocampus demonstrated an inverted U-shaped dose-response pattern (Fig. 3).

Effect of WSKY on $p E R K / E R K$ and $p C R E B / C R E B$ in the hippocampus. To investigate whether the hippocampal $\mathrm{BDNF} /$ TrkB downstream cascade, including activation of ERK (ERK1 and ERK2) and CREB, was involved with WSKY-induced SLM changes in rats, western blot analysis was used to measure the phosphorylation levels of ERK1/2 and CREB in the hippocampi of rats that were not subjected to behavioral tests. The rats receiving WSKY treatment at doses of 100 or $250 \mathrm{mg} / \mathrm{kg}$ for 14 days demonstrated significantly increased levels of BDNF in the hippocampus (WSKY $100 \mathrm{mg} / \mathrm{kg}, \mathrm{P}<0.001$; WSKY $250 \mathrm{mg} / \mathrm{kg}, \mathrm{P}<0.001$, compared with the control group, respectively; Fig. 4). The levels of phosphorylation of extracellular signal-regulated kinase (ERK1/2), which is an important component of the mitogen-activated protein kinase (MAPK)/ERK pathway, were significantly higher in the hippocampus (WSKY $100 \mathrm{mg} / \mathrm{kg}$, pERK1 P<0.001, pERK2 P<0.001; WSKY 250 mg/kg, pERK1 $\mathrm{P}<0.001$, pERK2 $\mathrm{P}<0.001$, compared with the control group, respectively). In addition, the activation of CREB was analyzed, which is phosphorylated at Ser-133 by ERK following ERK phosphorylation, and has a critical role in LTP and memory formation. The 14-day $250 \mathrm{mg} / \mathrm{kg}$ WSKY treatment produced increased levels of pCREB in the hippocampus (WSKY $100 \mathrm{mg} / \mathrm{kg}$, pCREB P<0.05; WSKY $100 \mathrm{mg} / \mathrm{kg}$, pCREB $\mathrm{P}>0.05$, compared with the control group, respectively; Fig. 4). These results suggested that WSKY improved the cognitive performance in the MWM test and may therefore be involved
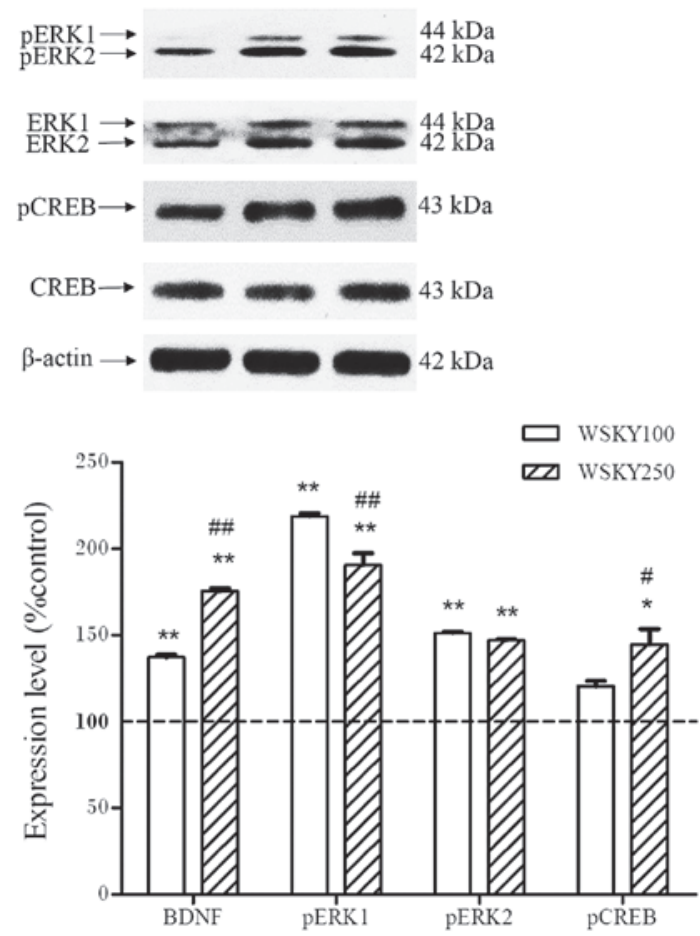

Figure 4. Effect of WSKY on hippocampal BDNF and BDNF downstream signaling components of rats without MWM test. Western blot analysis of BDNF, pERK, ERK, pCREB and CREB levels. Following treatment with WSKY, BDNF was significantly increased in the hippocampus compared with the control group. In addition, WSKY treatment increased levels of hippocampal pERK1/2, and its downstream signaling molecule pCREB. Quantification of BDNF data was normalized against $\beta$-actin, whereas pERK and pCREB were normalized against each unphosphorylated form and expressed as a percentage of the control (baseline). The blots represent typical results from three independent experiments. Densitometric data are presented as the mean \pm standard error of the mean. ${ }^{*} \mathrm{P}<0.05,{ }^{* *} \mathrm{P}<0.01$ vs. the control group; ${ }^{\#} \mathrm{P}<0.05,{ }^{\# \#} \mathrm{P}<0.01$ vs. the WSKY $100 \mathrm{mg} / \mathrm{kg}$ group (each group, $n=6$ ). WSKY, warm-supplementing kidney yang; BDNF, brain derived neurotrophic factor; MWM, Morris water maze; pCREB, phosphorylated cAMP response element binding protein; ERK, extracellular signal-regulated kinase.

in the upregulation of BDNF expression and activation of its downstream cascade.

\section{Discussion}

The cognitive deficits of schizophrenia, which are considered a core manifestation and an important predictor of the functional outcome, may be understood in the context of the molecular and cellular mechanisms of learning and memory, in which BDNF has a key role through the regulation of synaptic plasticity (17). Therefore, BDNF may be assumed to be a potential biomarker candidate, as it is implicated in cognitive impairment in schizophrenia. In the present study, the distinct effects of different doses of WKSY on cognitive performance in a chronic MK-801-induced rat model of schizophrenia were investigated. Numerous studies have demonstrated that acute or chronic treatment with NMDA antagonists, including phencyclidine (PCP) and MK-801, induced NMDA receptor (NMDAR) hypofunction and produced behavior in healthy patients which resembled psychotic symptoms, cognitive deficits and negative symptoms of schizophrenia (18). In the present study, rats received a total of 14 consecutive MK-801 
injections, as repeated treatment with MK-801 has been suggested as a better animal model of schizophrenia than a single dose $(19,20)$. Chronic MK-801 treatment impairs diverse factors in the working memory of rats, including attention, speed of processing, visual learning, long-term memory, reasoning and problem solving skills (21). In the acquisition phase, it was identified that MK-801 treatment increased the escape latency compared with that of the control group, and this impairment was rescued by a 14-day administration of WSKY doses of 25, 100 and $250 \mathrm{mg} / \mathrm{kg}$; however, without changing the swimming speed of the animals. In the probe test, $250 \mathrm{mg} / \mathrm{kg}$ WSKY treatment increased the time spent in the target quadrant and the number of platform crossings. This indicated that $250 \mathrm{mg} / \mathrm{kg}$ WSKY treatment ameliorated the MK-801-induced SLM impairment in the MWM test by improving murine ability of learning and enhancing consolidation of recognition memory.

The present study also investigated effects of WSKY on BDNF expression in the hippocampus of rat without training. NMDA antagonists, including PCP and MK-801, have been shown to induce cognitive impairment via inhibition of BDNF secretion and activation $(22,23)$. Therefore, the present study examined whether the underlying mechanism of the enhancement of SLM by WSKY was associated with the upregulation of BDNF in the hippocampal region. Hippocampal tissues were collected immediately following the last administration of WSKY. It was identified that a 14-day administration of WSKY at doses of 100,250 or $500 \mathrm{mg} / \mathrm{kg}$ significantly stimulated endogenous BDNF expression in the hippocampal region when compared with the MK-801-treated group. It is known that activation of TrkB by BDNF stimulates intracellular signaling cascades involved in plasticity, including the ERK/MAPK pathway and the PI3K/Akt pathway (24). Callaghan and Kelly (25) reported that the consolidation of recognition memory was associated with increased release of BDNF in the dendate gyrus (DG) and perirhinal cortex, which was associated with significant increases in ERK activation and c-fos expression in the hippocampal DG, and PI3K activation and c-fos expression in the perirhinal cortex in rats. Therefore, the present study aimed to confirm whether WKSY induced increases in levels of hippocampal BDNF expression, to clarify whether it activates the TrkB downstream signaling cascade. The expression levels of pERK and pCREB, which are important molecules involved in the BDNF/TrkB signaling pathway, were assessed by western blot analysis. It was identified that $250 \mathrm{mg} / \mathrm{kg}$ WSKY treatment significantly upregulated the phosphorylation of ERK1/2 as well as the phosphorylation of CREB in the hippocampus when compared with the vehicle-treated group. The ERK signaling pathway is involved in neuroplasticity and memory formation $(26,27)$, and the activation of the CREB signaling pathway in the hippocampus has an important role in spatial memory formation (28). The results suggested that WSKY may improve cognition via activation of the BDNF/TrkB downstream signaling cascade.

Of note, in the WSKY-treated groups, the effect of memory enhancement in the MWM test and the hippocampal BDNF protein expression levels both demonstrated an inverted U-shaped dose-response curve fashion. It has been suggested that aconitum alkaloids, including aconitine, lappaconitine and 6-benzoylheteratisine, three chemical components of
Radix Aconiti Lateralis, an ingredient of the WSKY formula, which is associated with $\mathrm{Na}^{+}$channels, have been shown to exert inhibitory effects on neuronal activity in rat hippocampal slices in vitro at specific doses $(29,30)$. It is therefore hypothesized that the inverted U-shaped dose-response curve of WSKY may be due to the inhibitory effect of aconitum alkaloids at high doses of WSKY. Further studies are required to clarify these issues.

In the present study, the effect of WSKY on schizophrenia-associated cognitive impairment by modulating BDNF expression and the BDNF/ERK/CREB pathway in hippocampus were elucidated, and it was identified that WSKY may be a potential cognitive enhancer for a number of psychiatric diseases, including schizophrenia. However, as BDNF has an important and unique role in regulating a wide range of functions at different neural development stages, and human epidemiology provides compelling evidence that exposure of the neonate, from the gestation or the perinatal period, to environmental adversities, including abnormal BDNF expression increase the risk of developing schizophrenia $(31,32)$. Considering the neurodevelopmental hypothesis of schizophrenia, BDNF has been proposed to be involved, at least in part, in the pathogenesis of this disease. Therefore, further studies are required to clarify the effect of WSKY on BDNF expression using neurodevelopmental schizophrenic animal models, including the disruption of neurogenesis during a critical gestational period, neonatal ventral hippocampal lesions and post-weaning social isolation. In addition, it is necessary to specify the effective components in WSKY to clear its mechanisms of action and develop more effective cognitive remediation for psychiatric diseases.

In conclusion, the results of the present study indicated that WSKY enhanced cognitive performance in schizophrenia by upregulating BDNF/ERK/CREB signaling in the hippocampus, and WSKY has potential therapeutic implications for cognitive impairment of schizophrenia.

\section{Acknowledgements}

The present study was supported by two grants from the Key Technology Research of Major Mental Illness Prevention and Treatment for the Barriers to the Recognition and Prevention of Depression and Anxiety in General Hospital (grant no. 2012BAIO1B00), and the Chinese Medicine research projects of Hubei Provincial Health Department, China (grant no. 2012Z-Y37). The authors also acknowledge the technical support from the HuBei Provincial Key Library of Digestive Disease and the Central Laboratory of Renmin Hospital of Wuhan University.

\section{References}

1. Taylor MA and Abrams R: Cognitive impairment in schizophrenia. Am J Psychiatry 141: 196-201, 1984.

2. Reichenberg A, Weiser M, Caspi A, et al: Premorbid intellectual functioning and risk of schizophrenia and spectrum disorders. J Clin Exp Neuropsychol 28: 193-207, 2006.

3. Kurtz MM, Moberg PJ, Gur RC and Gur RE: Approaches to cognitive remediation of neuropsychological deficits in schizophrenia: a review and meta-analysis. Neuropsychol Rev 11: 197-210, 2001. 
4. Lieberman JA, Stroup TS, McEvoy JP, et al: Effectiveness of antipsychotic drugs in patients with chronic schizophrenia. N Engl J Med 353: 1209-1223, 2005.

5. Marder SR and Fenton W: Measurement and Treatment Research to Improve Cognition in Schizophrenia: NIMH MATRICS initiative to support the development of agents for improving cognition in schizophrenia. Schizophr Res 72: 5-9, 2004.

6. Chen ZH, Wang GH, Wang XP, et al: Effects of warm-supplementing kidney yang (WSKY) capsule added on risperidone on cognition in chronic schizophrenic patients: a randomized, double-blind, placebo-controlled, multi-center clinical trial. Hum Psychopharmacol 23: 465-470, 2008.

7. ChenZH,WangGH,WangXP,etal:EffectofWarm-Supplementing Kidney Yang (WSKY) added to risperidone on quality of life in patients with schizophrenia: a randomized controlled trial. Clin Rehabil 23: 963-972, 2009.

8. Lee YS and Silva AJ: The molecular and cellular biology of enhanced cognition. Nat Rev Neurosci 10: 126-140, 2009.

9. Lipsky RH and Marini AM: Brain-derived neurotrophic factor in neuronal survival and behavior-related plasticity. Ann NY Acad Sci 1122: 130-143, 2007.

10. Buckley PF, Pillai A and Howell KR: Brain-derived neurotrophic factor: findings in schizophrenia. Curr Opin Psychiatry 24 $122-127,2011$

11. Favalli G, Li J, Belmonte-de-Abreu P, Wong AH and Daskalakis ZJ: The role of BDNF in the pathophysiology and treatment of schizophrenia. J Psychiatr Res 46: 1-11, 2012.

12. Ahlander M, Misane I, Schött PA and Ogren SO: A behavioral analysis of the spatial learning deficit induced by the NMDA receptor antagonist MK-801 (dizocilpine) in the rat. Neuropsychopharmacology 21: 414-426, 1999.

13. Reagan-Shaw S, Nihal M and Ahmad N: Dose translation from animal to human studies revisited. FASEB J 22: 659-661, 2008.

14. Morris R: Developments of a water-maze procedure for studying spatial learning in the rat. J Neurosci Methods 11: 47-60, 1984.

15. Niitsu T, Shirayama Y, Matsuzawa D, et al: Associations of serum brain-derived neurotrophic factor with cognitive impairments and negative symptoms in schizophrenia. Prog Neuropsychopharmacol Biol Psychiatry 35: 1836-1840, 2011.

16. Asevedo E, Gadelha A, Noto C, et al: Impact of peripheral levels of chemokines, BDNF and oxidative markers on cognition in individuals with schizophrenia. J Psychiatr Res 47: 1376-1382 2013.

17. Martinotti G, Di Iorio G, Marini S, et al: Nerve growth factor and brain-derived neurotrophic factor concentrations in schizophrenia: a review. J Biol Regul Homeost Agents 26: 347-356, 2012.

18. Gilmour G, Dix S, Fellini L, et al: NMDA receptors, cognition and schizophrenia - testing the validity of the NMDA receptor hypofunction hypothesis. Neuropharmacology 62: 1401-1412, 2012.
19. Mandillo S, Rinaldi A, Oliverio A and Mele A: Repeated administration of phencyclidine, amphetamine and MK-801 selectively impairs spatial learning in mice: a possible model of psychotomimetic drug-induced cognitive deficits. Behav Pharmacol 14: 533-544, 2003.

20. Li JT, Su YA, Guo CM, et al: Persisting cognitive deficits induced by low-dose, subchronic treatment with MK-801 in adolescent rats. Eur J Pharmacol 652: 65-72, 2011.

21. Mouri A, Nagai T, Ibi D and Yamada K: Animal models of schizophrenia for molecular and pharmacological intervention and potential candidate molecules. Neurobiol Dis 53: 61-74, 2013.

22. Castren E, da Penha Berzaghi M, Lindholm D and Thoenen H: Differential effects of MK-801 on brain-derived neurotrophic factor mRNA levels in different regions of the rat brain. Exp Neurol 122: 244-252, 1993

23. Adachi N, Numakawa T, Kumamaru E, et al: Phencyclidine-induced decrease of synaptic connectivity via inhibition of BDNF secretion in cultured cortical neurons. Cereb Cortex 23: 847-858, 2013

24. Bekinschtein P, Cammarota M, Igaz LM, et al: Persistence of long-term memory storage requires a late protein synthesis-and BDNF- dependent phase in the hippocampus. Neuron 53: 261-277, 2007.

25. Callaghan CK and Kelly ÁM: Differential BDNF signaling in dentate gyrus and perirhinal cortex during consolidation of recognition memory in the rat. Hippocampus 22: 2127-2135, 2012.

26. Ying SW, Futter M, Rosenblum K, et al: Brain-derived neurotrophic factor induces long-term potentiation in intact adult hippocampus: requirement for ERK activation coupled to CREB and upregulation of Arc synthesis. J Neurosci 22 1532-1540, 2002.

27. Mazzucchelli C and Brambilla R: Ras-related and MAPK signalling in neuronal plasticity and memory formation. Cell Mol Life Sci 57: 604-611, 2000.

28. Pláteník J J, Kuramoto N and Yoneda Y: Molecular mechanisms associated with long-term consolidation of the NMDA signals. Life Sci 67: 335-364, 2000.

29. Ameri A, Gleitz J and Peters T: Inhibition of neuronal activity in rat hippocampal slices by Aconitum alkaloids. Brain Res 738: 154-157, 1996.

30. Ameri A, Gleitz J and Peters T: Aconitine inhibits epileptiform activity in rat hippocampal slices. Naunyn Schmiedebergs Arch Pharmacol 354: 80-85, 1996.

31. Neill JC, Barnes S, Cook S, et al: Animal models of cognitive dysfunction and negative symptoms of schizophrenia: focus on NMDA receptor antagonism. Pharmacol Ther 128: 419-432, 2010.

32. Rapoport JL, Addington AM, Frangou S and Psych MR: The neurodevelopmental model of schizophrenia: update 2005. Mol Psychiatry 10: 434-449, 2005. 